\title{
Current situation of scrub typhus in South Korea from 2001-2013
}

\author{
Hyeong-Woo Lee ${ }^{1}$, Pyo Yun Cho², Sung-Ung Moon ${ }^{3}$, Byoung-Kuk Na ${ }^{4}$, Yoon-Joong Kang ${ }^{5}$, Youngjoo Sohn ${ }^{6}$, \\ Seung-Ki Youn ${ }^{7}$, Yeongseon Hong ${ }^{8}$ and Tong-Soo Kim²
}

\begin{abstract}
Background: The bacteria Orientia tsutsugamushi is the causative agent of scrub typhus, mite-borne disease, which causes an acute febrile illness in patients. An epidemiologic study was conducted to understand the characteristics of scrub typhus in South Korea.

Findings: Reporting of tsutsugamushi disease is mandatory in South Korea since 1994. To investigate the prevalence of tsutsugamushi disease from 2001 to 2013, medical records from the Korea Center for Disease Control and Prevention were reviewed. In total, 70,914 cases were reported during 2001-2013. Of these, 37.16\% (26,349) were male and 62.84\% $(44,565)$ were female. The highest number of cases was in the 60-69-year-old age group $(19,484 ; 27.48 \%)$, and $72.22 \%$ $(51,212)$ were in the $50-79$-year-old age group. There were 65,100 cases $(91.80 \%)$ reported during October $(24,964 ; 35.20 \%)$ and November $(40,136 ; 56.60 \%)$. An almost four-fold increase in the number of patients was observed in 2013 (10,485 cases) compared to 2001 (2,637 cases). The highest number of patients was reported in the Jeonbuk $(9,425 ; 13.29 \%)$ and lowest in the Jeju $(362 ; 0.51 \%)$.
\end{abstract}

Conclusions: A rapid increase in the incidence of patients with tsutsugamushi disease was observed in most areas from 2001 to 2013, with the majority of cases reported in the western and southern coast.

Keywords: Female, Incidence, Mites, Orientia tsutsugamushi, Republic of Korea, Scrub typhus, Trombiculidae

\section{Findings}

\section{Background}

Scrub typhus, tsutsugamushi disease, or chigger-borne rickettsiosis is an acute, febrile infectious disease among humans that is caused by infection with the bacterium Orientia tsutsugamushi following the bite of infected mite vectors. It is prevalent in the Asia-Pacific region, where about 1 million cases are reported annually and about 1 billion people may be at risk [1,2]. In South Korea, scrub typhus was first reported in six cases during the Korean War among United Nations military personnel [3], however, tsutsugamushi disease was unfamiliar to Koreans until 1986 when some suspected patients were diagnosed with tsutsugamushi disease. After that, scrub typhus was recognized as endemic in South Korea $[4,5]$, and since then, the disease has been one of the most common rickettsial diseases with poorly understood

\footnotetext{
* Correspondence: tongsookim@inha.ac.kr

${ }^{2}$ Departments of Parasitology and Tropical Medicine, Inha University School of Medicine, Incheon 400-712, Republic of Korea

Full list of author information is available at the end of the article
}

reasons and then become a major public health problem to farmers during harvest season. In 1994, scrub typhus was designated a Group III notifiable infectious disease by the Korea Centers for Disease Control and Prevention (KCDC). Subsequently, approximately 300 cases were reported annually, with a peak incidence of 6,562 cases in 2005 and a plateau pattern thereafter [6,7]. Since 2012, however, disease incidence has rapidly increased. Here, we report on the epidemiology of scrub typhus in South Korea in the last decade in order to enhance understanding of its geographic, temporal and demographic characteristics.

\section{Methods}

Data from the National Infectious Disease Surveillance (NIDS) system were used to analyze the epidemiology of scrub typhus in South Korea. The geographic distribution of the disease was determined based on patient residence at the time of diagnosis. The annual prevalence rate (APR: the number of cases per 100,000 people) was used to further analyze its geographic distribution. The disease's seasonal incidence was determined by grouping 
cases in monthly intervals. After obtaining approval from the Institutional Review Board of the Korean National Institute of Health, scrub typhus case records submitted to the KCDC were reviewed without revealing patient identity, and trends based on patients' age, sex, and occupation were analyzed.

\section{Results and discussion}

Analysis of the NIDS data revealed 70,914 cases of scrub typhus in South Korea from 2001 to 2013. Fewer than 2,700 cases were reported annually from 2001 to 2003, after which the incidence rapidly rose to a small peak of 6,780 cases in 2005, and then settled into a plateau pattern with a fluctuating number of annual indigenous cases. However, in 2012 and 2013 the incidence rapidly increased, with 10,485 cases reported in 2013 (Figure 1A).

The incidence of scrub typhus varies with sex, age, and occupation. Scrub typhus patients were more likely to be female $(44,565$ cases, $62.84 \%)$ than male $(26,349$ cases, $37.16 \%$ ). The sex-specific incidence (female/male ratio) did not change over time, varying slightly between 1.56 and 1.91 per year. Patients aged 60-69 years $(19,484$ cases) comprised the highest number of cases, followed by those aged 70-79 (16,141 cases) and 50-59
(15,587 cases) years. These age groups comprised $72.2 \%$ of the total number of scrub typhus cases (Figure 1A, Table 1). It is worth noting, however, that the disease has been increasing in all age groups, especially in children aged less than 10 years. Farmers had a higher incidence of scrub typhus than non-farmers, although the proportion of cases in non-farmers increased over the study period. The majority (91.8\%) of cases occurred in October and November, but a considerable number were also reported in December (3,995 cases, 5.6\%). Lowest cases $(75$ cases, $0.1 \%)$ were reported in March. Interestingly, 389 cases $(0.5 \%)$ were reported between January and February, i.e. the winter season in South Korea (Table 2).

Cases of scrub typhus were not evenly distributed throughout the country. It showed that the disease spread over time was more prevalent in rural areas than in urban areas. The highest number of cases was reported in Jeonbuk Province (JB, 9,425 cases; APR, 40.23) followed by Jeonnam Province (JN, 7,403 cases; APR, 32.14), Chungnam Province (CN, 8,493 cases; APR, 30.73), and Gyeongnam Province (GB, 8,746 cases; APR, 20.82) (Figure 1B). The primary industry in these provinces is agriculture or agriculture-related activities, and most

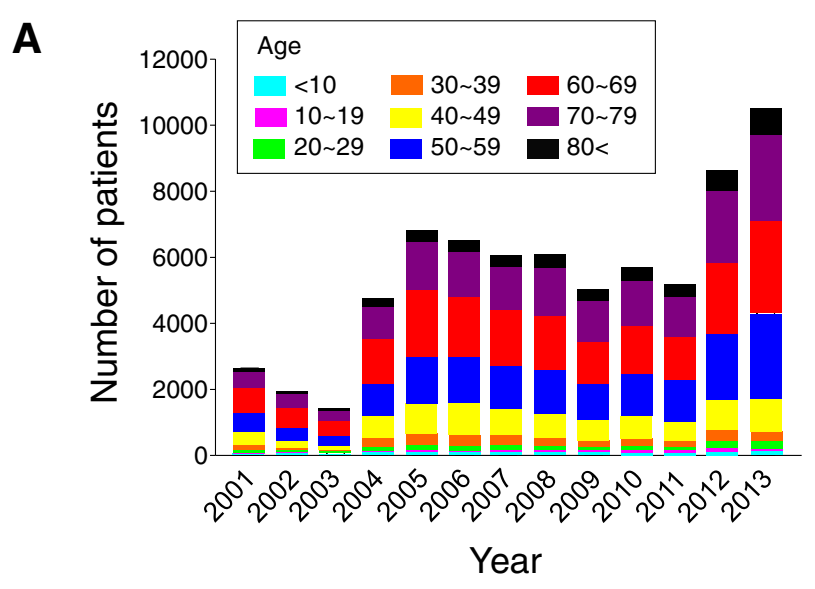

B

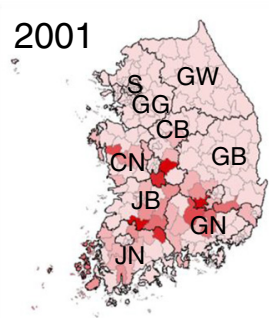

JT

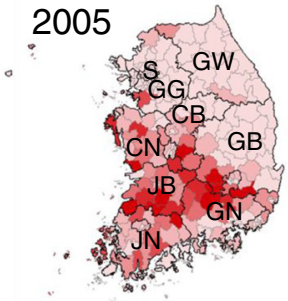

JS

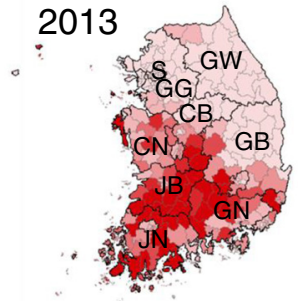

ST)

Figure 1 Scrub typhus in South Korea. (A) Total incidence of scrub typhus in South Korea between 2001-2013. (B) Annual prevalence rate and space-time clustering of scrub typhus cases at the county level in South Korea. SE, Seoul; GG, Gyeonggi Province; GW, Gangwon Province; CB, Chungbuk Province; CN, Chungnam Province; JB, Jeonbuk Province; JN, Jeonnam Province; GB, Gyeongbuk Province; GN, Gyeongnam Province; JJ, Jeju Province. 
Table 1 The number of Scrub typhus cases according to age in South Korea during 2001-2013

\begin{tabular}{lllllllllllllll}
\hline & $\mathbf{2 0 0 1}$ & $\mathbf{2 0 0 2}$ & $\mathbf{2 0 0 3}$ & $\mathbf{2 0 0 4}$ & $\mathbf{2 0 0 5}$ & $\mathbf{2 0 0 6}$ & $\mathbf{2 0 0 7}$ & $\mathbf{2 0 0 8}$ & $\mathbf{2 0 0 9}$ & $\mathbf{2 0 1 0}$ & $\mathbf{2 0 1 1}$ & $\mathbf{2 0 1 2}$ & $\mathbf{2 0 1 3}$ & Total \\
\hline$<10$ & 44 & 38 & 16 & 55 & 71 & 62 & 49 & 45 & 49 & 60 & 57 & 96 & 92 & 734 \\
$10-19$ & 26 & 15 & 20 & 55 & 79 & 69 & 76 & 61 & 64 & 77 & 79 & 124 & 111 & 856 \\
$20-29$ & 76 & 36 & 30 & 105 & 152 & 148 & 147 & 121 & 98 & 133 & 117 & 202 & 226 & 1,591 \\
$30-39$ & 168 & 108 & 62 & 297 & 372 & 350 & 320 & 259 & 215 & 250 & 205 & 444 & 379 & 3,429 \\
$40-49$ & 407 & 230 & 171 & 661 & 896 & 963 & 795 & 735 & 600 & 670 & 546 & 888 & 983 & 8,545 \\
$50-59$ & 556 & 366 & 265 & 928 & 1,408 & 1,378 & 1,281 & 1,320 & 1,076 & 1,259 & 1,231 & 1,970 & 2,549 & 15,587 \\
$60-69$ & 760 & 653 & 474 & 1,375 & 2,022 & 1,806 & 1,701 & 1,662 & 1,315 & 1,473 & 1,308 & 2,164 & 2,771 & 19,484 \\
$70-79$ & 478 & 387 & 295 & 973 & 1,419 & 1,348 & 1,303 & 1,454 & 1,237 & 1,349 & 1,241 & 2,099 & 2,558 & 16,141 \\
$80-89$ & 115 & 84 & 80 & 239 & 341 & 332 & 335 & 374 & 321 & 368 & 349 & 584 & 764 & 4,286 \\
$90>$ & 7 & 2 & 2 & 10 & 20 & 24 & 15 & 26 & 20 & 32 & 18 & 33 & 52 & 261 \\
Total & 2,637 & 1,919 & 1,415 & 4,698 & 6,780 & 6,480 & 6,022 & 6,057 & 4,995 & 5,671 & 5,151 & 8,604 & 10,485 & 70,914 \\
\hline
\end{tabular}

patients were farmers. Since most cases of scrub typhus occur in elderly farmers, the higher incidence of female patients may be due to differences in work behavior between men and women during the harvest season, when there is an increased possibility of exposure to infected chigger mites. Cases of scrub typhus have also increased in urban and suburban areas, correlating with the increase in the incidence of the disease in non-farmers. For instance, the APR in Seoul Metropolitan City increased from 0.54 in 2003 to 3.75 in 2012, in Busan Metropolitan City from 1.50 in 2003 to 23.30 in 2013, in Daegu Metropolitan City from 1.01 in 2003 to 19.54 in 2005, in Incheon Metropolitan City from 0.25 in 2002 to 4.44 in 2012, in Gwangju Metropolitan City from 3.45 in 2003 to 32.93 in 2013, in Daejeon Metropolitan City from 2.03 in 2003 to 33.86 in 2013, and in Ulsan Metropolitan City from 2.81 in 2003 to 59.73 in 2013. In our previous study about the geographical distribution of scrub typhus vectors, a survey of larval trombiculid mites had been conducted during 2005 to 2007 by collecting wild small twice a year at 24 sites nationwide. The predominant mite species were Leptotrombidium pallidum (52.6\%), Leptotrombidium scutellare (27.1\%), Leptotrombidium palpale (8.2\%), Leptotrombidium orientale (5.6\%), and Neotrombicula tamiyai (1.7\%). However, the geographical distribution map of the $L$. scutellare chigger index was identical to the incidence pattern of scrub typhus, whereas those of overall mites and L. pallidum showed no relationship with case incidence patterns [8].

The recent expansion of scrub typhus in South Korea may be closely associated with factors such as the physical environment and human activities. Future research is required to identify the role these factors play in the epidemiology of the disease. It is expected that the number of scrub typhus cases will continue to increase, although there may be some fluctuations. An intensive nationwide

Table 2 The number of Scrub typhus cases by month in South Korea during 2001-2013

\begin{tabular}{|c|c|c|c|c|c|c|c|c|c|c|c|c|c|}
\hline & Jan & Feb & Mar & Apr & May & Jun & Jul & Aug & Sep & Oct & Nov & Dec & Total \\
\hline 2001 & 5 & 1 & 1 & 1 & 1 & 0 & 4 & 4 & 7 & 1,007 & 1,499 & 107 & 2,637 \\
\hline 2002 & 6 & 3 & 3 & 1 & 7 & 7 & 3 & 5 & 22 & 962 & 843 & 57 & 1,919 \\
\hline 2003 & 5 & 3 & 2 & 4 & 7 & 6 & 3 & 1 & 9 & 642 & 642 & 91 & 1,415 \\
\hline 2004 & 7 & 1 & 2 & 2 & 6 & 6 & 0 & 6 & 14 & 1,829 & 2,667 & 158 & 4,698 \\
\hline 2005 & 10 & 2 & 0 & 3 & 4 & 9 & 7 & 5 & 24 & 2,159 & 4,167 & 390 & 6,780 \\
\hline 2006 & 11 & 3 & 4 & 13 & 8 & 9 & 5 & 5 & 29 & 2,054 & 3,766 & 573 & 6,480 \\
\hline 2007 & 25 & 10 & 6 & 11 & 13 & 17 & 12 & 11 & 26 & 1,123 & 4,374 & 394 & 6,022 \\
\hline 2008 & 14 & 10 & 2 & 8 & 22 & 8 & 12 & 6 & 38 & 3,035 & 2,547 & 355 & 6,057 \\
\hline 2009 & 39 & 17 & 13 & 15 & 14 & 21 & 14 & 12 & 51 & 1,739 & 2,641 & 419 & 4,995 \\
\hline 2010 & 23 & 13 & 11 & 16 & 27 & 27 & 27 & 27 & 60 & 1,203 & 3,832 & 405 & 5,671 \\
\hline 2011 & 37 & 11 & 10 & 13 & 25 & 24 & 18 & 28 & 77 & 1,825 & 2,718 & 365 & 5,151 \\
\hline 2012 & 51 & 41 & 15 & 17 & 20 & 14 & 25 & 36 & 102 & 3,680 & 4,341 & 262 & 8,604 \\
\hline 2013 & 25 & 16 & 6 & 9 & 31 & 33 & 40 & 27 & 74 & 3,706 & 6,099 & 419 & 10,485 \\
\hline Total & 258 & 131 & 75 & 113 & 185 & 181 & 170 & 173 & 533 & 24,964 & 40,136 & 3,995 & 70,914 \\
\hline
\end{tabular}


surveillance system is needed to ensure rapid diagnosis and treatment, and to monitor the changing environment, including the rodent population, and emerging drug resistant strains, until effective vaccine development reduces its incidence. In addition, education of the public through the media on the risk factors and symptoms of scrub typhus, together with personal protection methods during farming, gathering chestnuts, even outdoor activities during winter season, and taking breaks in areas adjacent to agricultural operations, should be undertaken.

\section{Competing interests}

The authors declare that they have no competing interests.

\section{Authors' contributions}

TSK and HWL conceived and designed the study and contributed to the execution of the research. TSK and HWL wrote the manuscript. SUM, YH, SKY, and BKN confirmed the scrub typhus cases. PYC, YS, YH, SKY, and YJK collected the patient information and performed the analysis. All authors have read and approved the final manuscript.

\section{Acknowledgments}

This work was supported by an Inha University Research Grant.

\section{Author details}

'Department of Pathology, Immunology, and Laboratory Medicine, College of Medicine, University of Florida, Gainesville, FL 32610, USA. ${ }^{2}$ Departments of Parasitology and Tropical Medicine, Inha University School of Medicine, Incheon 400-712, Republic of Korea. ${ }^{3}$ Department of Internal Medicine, Seoul National University Bundang Hospital, Seongnam 463-939, Korea.

${ }^{4}$ Department of Parasitology and Institute of Health Sciences, Gyeongsang National University School of Medicine, Jinju 660-751, Korea. ${ }^{5}$ Department of Biomedical Science, Jungwon University, Goesan, Chungbuk 367-805, Republic of Korea. ${ }^{6}$ Department of Anatomy, College of Korean Medicine, Institute of Korean Medicine, Kyung Hee University, Seoul 130-701, Korea. ${ }^{7}$ Department of Epidemiology, National Institute of Health, Osong 363-951, Republic of Korea. ${ }^{8}$ Department of Public Health, Sahmyook University, Seoul 139-742, Republic of Korea.

Received: 8 April 2015 Accepted: 13 April 2015

Published online: 19 April 2015

\section{References}

1. Watt G, Parola P. Scrub typhus and tropical rickettsioses. Curr Opin Infect Dis. 2003;16:429-36.

2. Kelly DJ, Fuerst PA, Ching WM, Richards AL. Scrub typhus: the geographic distribution of phenotypic and genotypic variants of Orientia tsutsugamushi. Clin Infect Dis. 2009;48 Suppl 3:S203-30.

3. Munro-Faure A, Missen MD. Scrub typhus in Korea. J R Army Med Corps. 1951;97:227-31.

4. Lee JS, Ahn C, Kim YK, Lee MH. Thirteen cases of rickettsial infection including 9 cases of tsutsugamushi disease first confirmed in Korea. J Korean Med Assoc. 1986;29:430-8.

5. Kim E, Park YS, Kim JM, Hong CS, Moon YM. Six cases of tsutsugamushi disease. Korean J Infect Dis. 1987;19:179-86.

6. Kweon SS, Choi JS, Lim HS, Kim JR, Kim KY, Ryu SY, et al. Rapid increase of Scrub typhus, South Korea, 2001-2006. Emerg Infect Dis. 2009;15:1127-9.

7. Jeong MA, Youn SK, Kim YK, Lee H, Kim SJ, Sohn A. Trends in the incidence of scrub typhus: the fastest growing vector-borne disease in Korea. Osong Public Health Res Perspect. 2013;4:166-9.

8. Roh JY, Song BG, Park WI, Shin EH, Park C, Park MY, et al. Coincidence between geographical distribution of Leptotrombidium scutellare and scrub typhus incidence in South Korea. PLoS One. 2014;12:9.

\section{Submit your next manuscript to BioMed Central and take full advantage of:}

- Convenient online submission

- Thorough peer review

- No space constraints or color figure charges

- Immediate publication on acceptance

- Inclusion in PubMed, CAS, Scopus and Google Scholar

- Research which is freely available for redistribution 